\title{
Interference in Wireless Networks: Causes, Analyses and Practical Mitigating Techniques
}

\author{
A. Hameed ${ }^{1} \&$ Ali Oudah ${ }^{1}$ \\ ${ }^{1}$ Faculty of Manufacturing Engineering, Universiti Malaysia Pahang (UMP), 26600 Pekan Pahang, Malaysia \\ Correspondence: A. Hameed, Faculty of Manufacturing Engineering, Universiti Malaysia Pahang (UMP), 26600 \\ Pekan Pahang, Malaysia. E-mail: multicore.processor@yahoo.com
}

\author{
Received: May 25, $2014 \quad$ Accepted: June 4, $2014 \quad$ Online Published: August 6, 2014 \\ doi:10.5539/mas.v8n5p56 URL: http://dx.doi.org/10.5539/mas.v8n5p56
}

\begin{abstract}
This paper addresses the key characteristics of interference in wireless networks. Moreover, it considers the impact of interference from Code Division Multiple Access base station to Wideband-Code Division Multiple Access one. A detailed discussion of major interference mitigation techniques in wireless networks, in general, and CDMA and W-CDMA systems, in particular is also imparted on. The techniques and findings in this paper can be easily used in the analyses of other wireless technologies.
\end{abstract}

Keywords: interference, spurious emissions, inter-modulations, mitigation techniques, guard band, isolation

\section{Introduction}

Coexistence of wireless systems in various frequency bands has become a commonplace (Shamsan et al. 2012). In a multi-operator situation and in an area where several network operators are striving to furnish mobile services, placing a cell site without interference is often a hurdle faced by operators (Roke Manor Research Ltd 2008). It is vital to realize the risk of interference between systems when planning in such an environment (Nguyen \& Zaghloul, 2007).

In circumstances where several mobile networks of different air interfaces operate in a specific province in a combination of frequency bands (i.e. $900,1800,1900$ and $2100 \mathrm{MHz}$ ), the probability of developing interference is inevitable (Oudah, 2013). This end-result is blended in territories where there is no site or districting standard and when there are many operators for each of the reserved frequency bands (Li \& Tatesh, 2009). In some regions, identical spectrum portions have been licensed to various operators in contiguous geographical domains. Indeed, it is a great obstacle to fight interference when the two systems operate in the same frequency band in neighboring service regions with non-collocated sites. In nations where siting constraints are widespread, particularly in urban and suburban areas, an operator is further restricted as controls are also put on the position of cell sites together with the corresponding antenna masts (Ball et al. 2008).

In occasions where the same frequency band is employed by several operators, interference may still be reduced while respecting siting stipulations, by sharing towers, antennas, etc.; however, additional obstacle is to create a tower that can fit a number of antennas while retaining the overall interference to a lowest (Kazemitabar, 2010). The installation of antennas at distinctive heights (vertical separation) for different frequency bands in disparate clutter types is an additional key consideration that can mitigate interference levels (ITU-R M.2039-2 2010).

The objective of this paper is to describe key features of interference in emerging cellular systems. Furthermore, the interference between Code Division Multiple Access (CDMA) and Wide-CDMA (WCDMA) technologies is analyzed and potential mitigation techniques are also discussed. To serve its purpose, this paper is organized in the following order: section two is an in-depth discussion of major interference causes and measures. Section three embarks on the analysis of interference between two coexisted systems, i.e. CDMA and W-CDMA, where potential interference issues are analyzed and discussed along with possible mitigation techniques. Section four concludes the findings.

\section{Interference Related Factors}

Separation between networks is interpreted as the attenuation between the transmitter reference point in the interfering unit, namely Base Station (BS) or Mobile Station (MS) and the receiver reference point (MS or BS). As shown in Fig. 1, it is likely that one BS could potentially disrupt another BS. The separation between the 
reference points in this case is given below:

$$
\begin{gathered}
\text { Available Isolation }=\text { Tx Feeder loss - Tx Antenna gain }+ \text { Propagation loss }- \text { Rx Antenna gain } \\
+ \text { Rx Feeder loss }+ \text { Extra attenuation provided by special filters }
\end{gathered}
$$

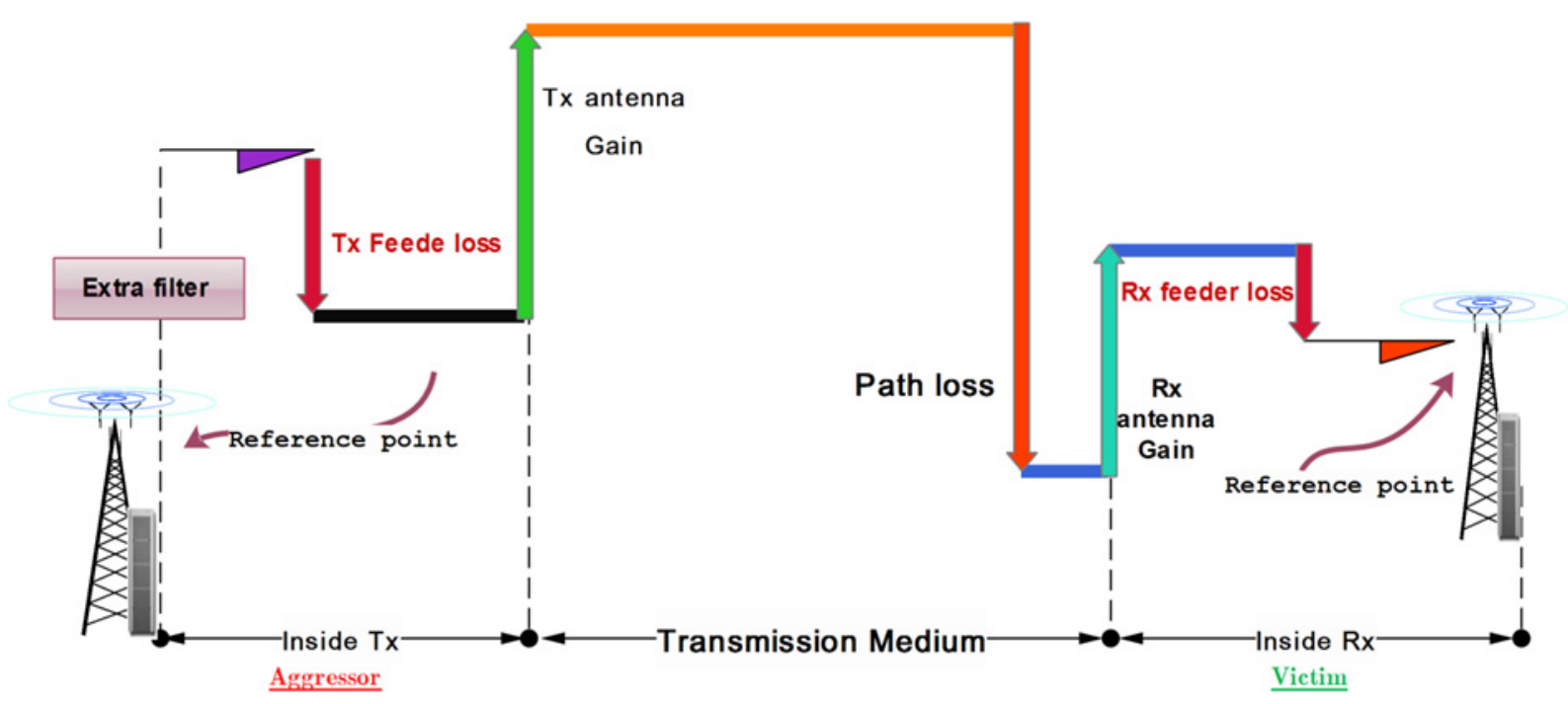

Figure 1. Transmission metrics between two adjacent systems

For gaps more than $10 \mathrm{~m}$, the path loss can be bordered to a free space pathloss model based on the ambience. For short antenna splits, the amount of the separation, i.e., (propagation loss - the combined transmitted and receiver antenna gains) may be approximated by evaluating coupling loss of the typical antennas. For extended separation, particular band pass filters are required.

Employing extra filters in transmit as well as receive paths, one can alleviate or at least lessen the impact of the interference that emerges owing to the coexistence of several wireless networks. The needed filter operation prerequisite can differ hugely relying on the operation frequency, bandwidth, attenuation condition and expense.

Other important elements in interference analysis are the so-called spurious emissions. These mostly give rise to all undesired transmissions from a transmitter. The emissions may incorporate inter-modulation (IM) products stimulated by various frequencies used internally in the Base Station transmitter, the inter-modulation induced by extrinsic carrier frequencies, the harmonics (peaks at double the carrier frequency) and the noise. All of these can result in a co-channel or adjacent channel interference to a neighboring victim receiver.

Interestingly, spurious emissions are sporadic transmissions outside the carrier frequency, but transmitter noise is the weakest level of successive wide-band emission. Transmitter noise cannot be reduced with radio frequency (RF) planning solely, as it is interrelated to the Noise Figure (NF) of the transmit link. This wide-band RF noise is also thought of as sideband noise. The majority of this noise is formed in the exciter section and magnified in following stages and ended at the transmitter output phases. Some transmitters are worse than others in forming this noise.

Furthermore, Inter-modulation (IM) products are established both in transmit and receive links, as two or more frequencies are combined and amplified in non-linear equipment. IM products of order ' $n$ ' are the sums and differences in ' $n$ ' terms of the primary frequencies. Typically, the greater the order of the IM product, the lesser is its strength. Furthermore, if one of the terms of the product is lower than the rest, it follows that the consequent IM product energy will also decline significantly. The imperfections of the equipment give rise to those unwanted frequencies that will result in a co-channel interference at the victim receiver. Normally, there are two major types of IM products: transmitted and receiver-generated IM(s). On one hand, the transmitter IM products are generated in the BS by mixing of carriers in the same power amplifier, connectors, duplex filter, combiner or the antenna. Transmitter IM can cause co-channel interference, which needs high separation between cells or additional filters to be used to in the intruding cell. Appending filters turns out to be challenging if the intruder cell belongs to a different operator. On the other hand, two or more electromagnetic waves can give rise to inter-modulation in the victim receiver featuring some imperfections. These inter-modulation waves generate 
interference if they are in the range of the receiver's bandwidth as they are received and amplified in a way similar to that of the wanted transmission. Accordingly, inter-modulation measures are essential in order to diagnose those surrounding transmitters that may impair the operation of the receiver owing to inter-modulation impact. Receiver (Rx) IM is created in the receiver amplifiers. The primary disparity between Tx IM and Rx IM with respect to co-existence is that the amount of co-channel interference from Tx IM is more conceivable than interference from Rx IM.

Remarkably, the intensity of Rx IM3 and IM5 rises 3 and 5 times as fast (in $\mathrm{dB}$ ) as the received carriers creating this IM. Given that the powers of Rx IM products step up quickly with the power of the strong received (undesired) carriers, Rx IM is often affiliated with a Rx IM rejection level. Carriers higher than the IM rejection level can lead to serious interference while carriers lower than the level are tolerable or harmless. Both IM3 and IM5 ought to be thought about as receiver IM interference. Usually, the IM behavior of a receiver is reported in terms of inter-modulation rejection ratio and is associated with the 3rd Inter-mod intercept point (IIP3) of the receiver interface. For instance, the Pin IM (intermodulation power in $\mathrm{dBm}$ ) equivalents to $3 \times \mathrm{Pi}-2 \times \mathrm{IIP} 3$ where $\mathrm{Pi}$ is the equal power in the two overlapping signals in $\mathrm{dBm}$ and IIP3 is the 3rd order Inter-mod intercept point of the receiver in $\mathrm{dBm}$. An additional filter in front of the influenced receiver stage can solve the dilemma.

Normally, receivers are devised to acknowledge particular forms of electromagnetic waves within the bounds of a fixed frequency band. Nevertheless, receivers additionally respond to unwanted signals featuring different modulation and frequency peculiarities. Occasionally, the interfering signals fall into one of the following fundamental types:

- Co-Channel Interference (CCI): This is due to emissions featuring frequencies that present within the bounds of the narrowest pass-band of the receiver.

- Adjacent Channel Interference (ACI): This is owing to undesired signals possessing frequency parts that exist within or nearby the receiver pass-band. Generally, adjacent channel interference is the interference between two wireless networks whose frequency carrier assignments are adjacent or contiguous, particularly in cases where their cell towers are not collocated.

- Out-of-band Interference (OOBI): This occurs when signals having frequency parts, that are outside of the receiver pass-band. If this interference overtakes the noise floor of the victim receiver, it weakens the noise figure of the receiver. In order to combat this type of impairment, either the out-of-band signals must be to be decreased (by inserting filters to the interfering transmitter) or the desired signal level at the victim receiver be improved.

The receiver selectivity serves a significant purpose in attenuating adjacent channel interference. Furthermore, the intermediate frequency (IF) selectivity is the ability of a receiver to filter out any adjacent channels interference. The combination of the transmitter emission mask, the RF and IF selectivities together decide the frequency separation requirements.

Usually, receivers are subject to enormous out-of-band signals that give rise to a spurious response in the receiver. A spurious response can be initiated if the frequency of an intruding signal is such that the signal or one of its harmonics can merge with a local oscillator or one of its harmonics to output an IF in the receiver IF pass-band. The main crucial frequency in this respect is the image frequency of the receiver. In order to hold positive spurious response rejection, the sensitivity threshold of the image frequency should be considered. However, this causes detailed image frequency/spurious response qualities of the receiver if interference issues owing to image frequency are found.

A high interference level inside the RF bandwidth of a receiver can lead to interference even if the emission is outside the pass-band of the IF amplifiers resulting in a decrease of gain for the wanted signal owing to imperfections in the receiver front end. Such end-result gives rise to decreased Signal-to-Noise ratio $(\mathrm{S} / \mathrm{N})$ of the receiver, if a specific saturation reference power threshold is exceeded. This occurrence is ordinarily named blocking or desensitization. For this goal, the interference level at the front end of the receiver requires to be calculated over the whole RF bandwidth of the receiver. Namely, the receiver blocking or desensitization level is the highest level of a non-cochannel or adjacent channel interference that may be experienced as a low input signal (about three $\mathrm{dB}$ above the sensitivity level) without lowering the receiver operation.

Typically, noise, spurious emissions and transmitter IM products outside the allocated frequency block are inherent in any transmitter. These emissions give rise to noise and can stimulate interference to a neighboring receiver that is set to a weak signal from a transmitter (BS or MS) which is far away. As a rule, closer the interfering channel to the wanted channel, more responsive the receiver is to the interference. The interference is 
a combination of the adjacent channel interference due to deficient suppression in the receiver band-pass filter and the co-channel interference from the wide-band noise of the closer interfering transmitter. Fig. 2 depicts some of the elements explained above.

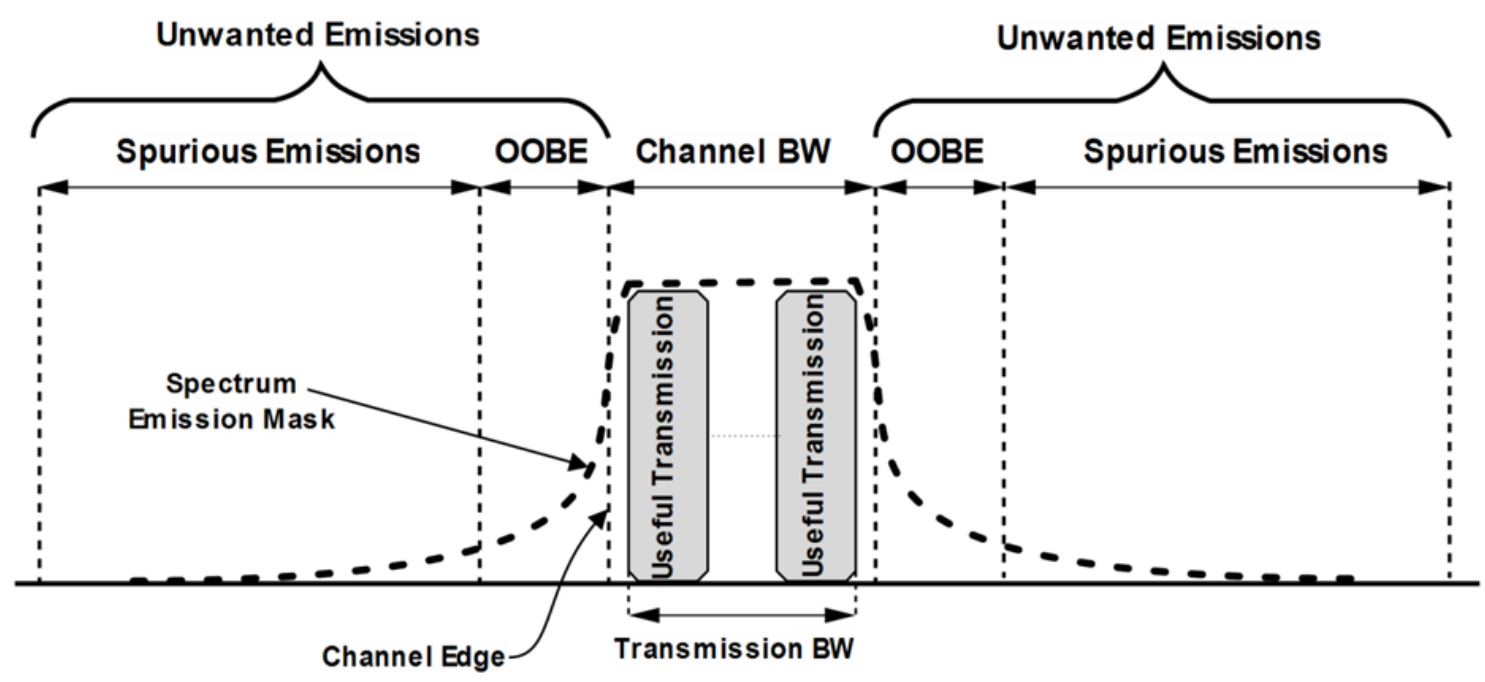

Figure 2. Key unwanted emissions in wireless systems

\section{Interference from CDMA to W-CDMA Networks}

In several countries in Asia, there has always been an argument as to whether Personal Communications Service (PCS) band (1850-1910/1930- $1990 \mathrm{MHz}$ ) allocations can coexist (fully or partially) with $2.1 \mathrm{GHz}$ band allocations, also referred to as the UMTS band (1920-1980/2100-2170 MHz) and if so, what are the acceptable degrees of interference and the needed guard bands between these blocks. There is $(60+60) \mathrm{MHz}$ of spectrum openness if allocated either for PCS or for UMTS solely. See Fig. 3 for another potential sharing of the spectrum between PCS and 2.1 GHz band allocations (ITU-R M.2116-1 2010).

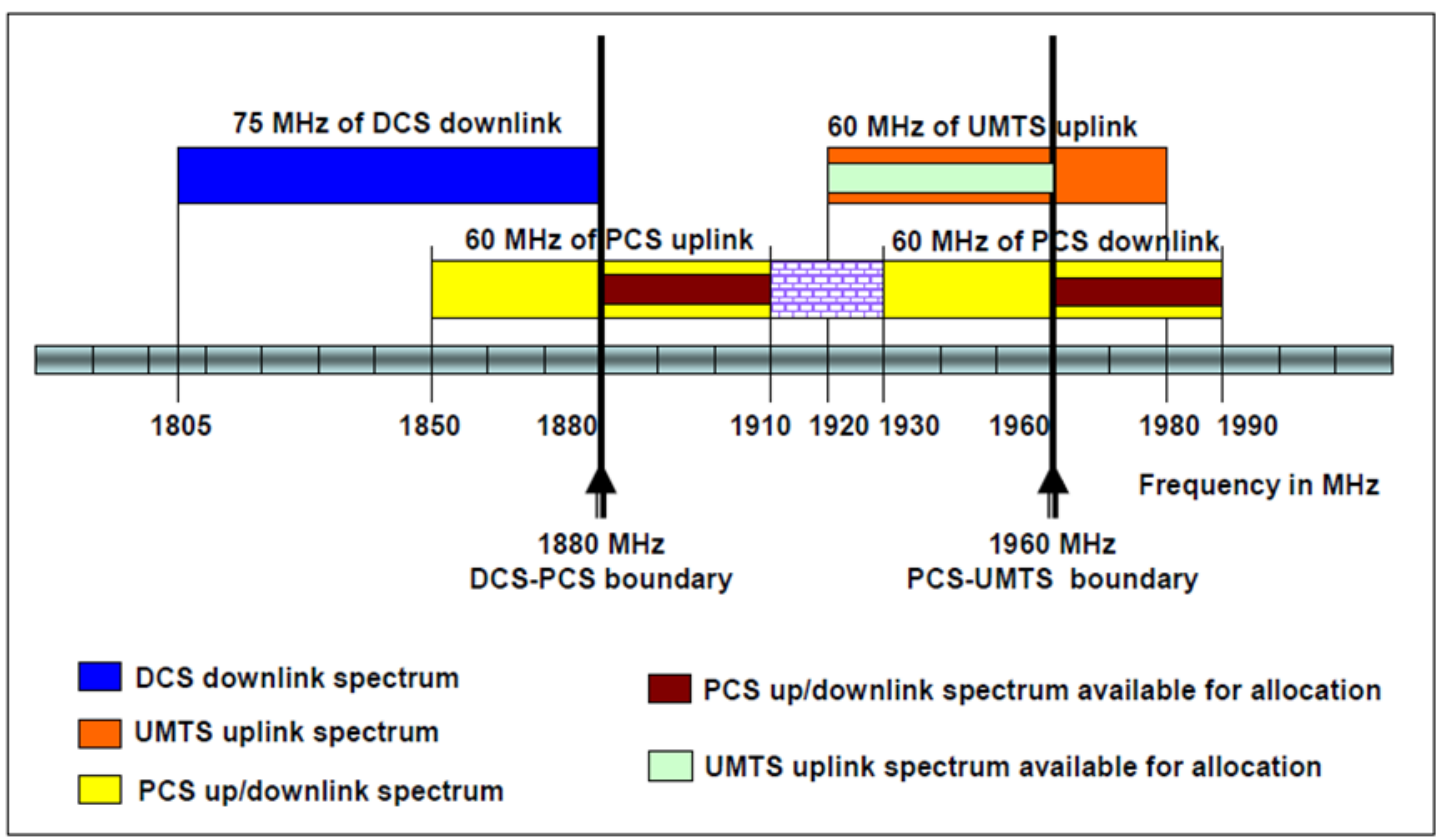

Figure 3. Potential spectrum sharing scenario between PCS and UMTS bands 
In this band sharing plan, if the operators with PCS band allocation are the holders, then the matter will be to protect the holders while assigning more spectrums in the $2.1 \mathrm{GHz}$ band. In some other situations, the conflict has been to rule out PCS allocations to protect forthcoming IMT-2000 core band allocations. In Fig. 3, it is depicted that the border between PCS and UMTS band allocations is $1960 \mathrm{MHz}$. With $5 \mathrm{MHz}$ protection band (from 1955 to $1960 \mathrm{MHz}$ ) at the $1960 \mathrm{MHz}$ margin, one gets $(30+30) \mathrm{MHz}$ for PCS and $(35+35) \mathrm{MHz}$ for UMTS operations. It is viable to move the border to either side of the spectrum (anywhere, from 1920 to 1980 $\mathrm{MHz}$ ) to earmark various bandwidth sharing between PCS and UMTS operations.

The next section assesses potential coexistence issues between CDMA2000 network in the PCS band and WCDMA network in the $2.1 \mathrm{GHz}$ band by scrutinizing the smallest guard band necessary between the two networks.

\section{Formulation, Results and Discussion}

In this subsection, the interference from CDMA2000 baste station transmitter to the UMTS Node-B receiver is considered. The Thermal Noise Floor at the UMTS Node-B receiver is found as follows:

$$
T N=-174 \frac{d B m}{H z} \times K \times T+10 \times \log (3.84)+N F_{R x}
$$

Where $T N$ is the thermal noise of UMTS receiver, $K$ is Boltzman's constant $=1.380 \times 10^{23} T$ is receiver's temperature and $N F_{R x}$ is UMTS receiver noise floor. For receiver noise figure of $5 \mathrm{~dB}, \mathrm{TN}=-103 \mathrm{dBm} / 3.84 \mathrm{MHz}$. As per 3GPP specification (3GPP TS 25.942), a maximum tolerable level of interference $\left(I_{R X}\right)$ from the intruder base station (Node-B) to the victim Node-B receiver is given as $-110 \mathrm{dBm} / 3.84 \mathrm{MHz}$ (3GPP TS 25.942). With $T N$ value of $-103 \mathrm{dBm} / 3.84 \mathrm{MHz}$ for WCDMA Node-B receivers, a spurious signal at the maximum tolerable limit of $-110 \mathrm{dBm} / 3.84 \mathrm{MHz}$ would be $7 \mathrm{~dB}$ below the $T N$ value and its presence would cause a receiver sensitivity degradation of $0.8 \mathrm{~dB}$, which is calculated as per the formula shown below (Oudah et al. 2012):

$$
S=10 \times \log \left(1+10^{\frac{I_{R x}-T N}{10}}\right)
$$

Where $S$ is the degradation in receiver sensitivity, $I_{R \mathrm{x}}$ is maximum tolerable level of interference and $T N$ is thermal noise. Similarly, 3GPP specifies a blocking requirement for WCDMA Node-B at a received spurious signal level of $-115 \mathrm{dBm} / 3.84 \mathrm{MHz}$ [10]. The processing gain $(P G)$ of the WCDMA signal is $25 \mathrm{~dB}$ (i.e., $10 \times \log$ $(3840000 / 12200))$ and for a target $\mathrm{Eb} / \mathrm{It}$ of $5 \mathrm{~dB}$, the Node-B receiver reference sensitivity would be of the order of $-123 \mathrm{dBm} / 3.84 \mathrm{MHz}$ (i.e., $T N+\mathrm{Eb} / \mathrm{It}-P G=-103+5-25=-123 \mathrm{dBm} / 3.84 \mathrm{MHz}$ ). In PCS band, the antenna gains are in the order of $15 \mathrm{dBi}$ and the feeder cable losses would be around $3 \mathrm{~dB}$. Hence, the equivalent isotropically radiated power (EIRP) of CDMA BS Tx would be around $55 \mathrm{dBm}(43+15-3=55 \mathrm{dBm})$.

Assuming that the interfering CDMA2000 BS in PCS band has a vendor specific spurious emission limit of - 75 $\mathrm{dBc} / 30 \mathrm{kHz}(20 \mathrm{~dB}$ better than the standards specified spurious emission limits of $-55 \mathrm{dBC} / 30 \mathrm{kHz})(3 \mathrm{GPP} T \mathrm{~S}$ 25.942) for a carrier-to-carrier separation greater than $1.98 \mathrm{MHz}$, the amount of required isolation $I_{\text {isolation }}(\mathrm{dB})$ achieved through spatial separation of antennas to mitigate the interference due to the out-of-band spurious emissions can be found as follows:

$I_{\text {isolation }}(\mathrm{dB})=T x \operatorname{EIRP}(\mathrm{dBm})-$ Tx out-of-band spurious emission limit - 10× $\log (30 / 1250)-3 \mathrm{GPP}$ specified WCDMA maximum tolerable level of Interference - Rx feeder cable loss (dB) + Rx antenna gain (dB) - Tx filter attenuation $(\mathrm{dB})$

$$
\begin{aligned}
& =55(\mathrm{dBm})-75+10 \times \log (1250 / 30)-(-110 \mathrm{dBm})-3(\mathrm{~dB})+15(\mathrm{dBi})-T x_{\text {filter-attenuation }} \\
& =(55-75+16+110-3+15)-T x_{\text {filter_attenuation }}(\mathrm{dB}) \\
& =118-T x_{\text {fitter_attenuation }}(\mathrm{dB})
\end{aligned}
$$

Therefore, with an extra filter of realistic complexity and cost in the CDMA BS Tx path with $60 \mathrm{~dB}$ attenuation, the required isolation due to physical separation of antennas " $I_{\text {isolation }}(\mathrm{dB})$ " $=118-60=58 \mathrm{~dB}$, which can very easily be realized by having about 40 meters of physical separation between CDMA2000 BS Tx and WCDMA Node-B Rx antennas. With some vertical separation of antennas, one can use lesser values for antenna gains and hence the burden on the required isolation further reduces.

As per 3GPP specification (3GPP TS 25.104), there is a $5 \mathrm{~dB}$ lower (more stringent) constraint on WCDMA Node- B tolerable blocking level compared to the maximum tolerable level for out-of-band Interference (- 115 $\mathrm{dBm}$ versus $-110 \mathrm{dBm}$ ), which translates directly into an extra $5 \mathrm{~dB}$ isolation requirement for blocking due to 
Intermodulation. Therefore, the isolation requirement with physical separation of antennas to mitigate blocking would be around $65 \mathrm{~dB}$. Hence, blocking would be the main issue for WCDMA Node-B.

It is worth mentioning that there are no clear requirements in band class 6 (IMT-2000) to protect WCDMA Node- B receiver in a co-area operation scenario from the spurious emissions caused by a CDMA2000 BS of another operator. But, most of the vendors would supply CDMA2000 BSs with spurious emissions that are most likely be considerably lower than the standards defined values, because a CDMA2000 BS needs to protect its receiver from its own transmitter.

As with CDMA2000 BS system, one can assume that the blocking performance of WCDMA Node-B receiver would most likely be expressively better than the standards stated value, because WCDMA Node-B system also has to protect its receiver from its own transmitter.

Using specified performance values for BS transmitters and receivers to establish isolation requirements also leads to overly pessimistic results, since they are not describing the real equipment performance. Accordingly, around $65 \mathrm{~dB}$ of isolation requirement is sufficient to meet the BS to Node-B spurious emission as well as intermodulation interference conditions, and it is quite possible to achieve such isolation requirement through physical separation of antennas with good antenna installation practices. $65 \mathrm{~dB}$ of antenna isolation is achievable with a site-to-site spacing of around 60 meters.

\section{Conclusions}

In this paper, deployment analysis of wireless systems has been discussed in details. The consequences of spurious emissions between two collocated systems and other related side-effects have also been discussed. It has been shown that analyses which looked into the coexistence of collocated CDMA2000 with WCDMA operating in the same geographic area are established on deterministic computations by supposing the worst-case scenario, i.e., BS /Node-B behavior according to minimum prerequisites stipulated in the related standard and maximum transmit power of BS /Node-B. Nevertheless, it is estimated that the real CDMA2000 BS out-of-band spurious emissions and blocking specifications of UMTS Node-B receivers are considerably better than the smallest requirements because CDMA2000 system needs to protect its receiver from its own transmitter. Consequently, the current equipment performance estimated to be at least $22 \mathrm{~dB}$ greater than the minimum performance specifications should be used. As a result, a minimum guard band of $5 \mathrm{MHz}$ is adequate assuming actual filters and separations resulting from practical antenna separation. In reality, the guard band between the UMTS uplink and the PCS downlink is constantly more than $5 \mathrm{MHz}$ as described below.

\section{Acknowledgment}

This research work has not been possible without the financial support from Universiti Malaysia Pahang (UMP) Vot. RDU 130387. Therefore, the authors would like to thank UMP for the continuous support.

\section{References}

Ball, C., Hindelang, T., \& Kambourov, I. (2008). Spectral efficiency assessment and radio performance comparison between LTE and WiMAX (pp. 0-5). Indoor and Mobile Radio.

ITU-R M.2039-2. (2010). Characteristics of terrestrial IMT-2000 systems for frequency sharing/interference analyses.

ITU-R M.2116-1. (2010). Characteristics of broadband wireless access systems operating in the land mobile service for use in sharing studies.

Kazemitabar, S. J. (2010). Coping with Interference in Wireless Networks (1st ed.). Springer.

Li, J., \& Tatesh, S. (2009). Coexistence studies for 3GPP LTE with other mobile systems. IEEE Communications Magazine, 47(4), 60-65. http://dx.doi.org/10.1109/MCOM.2009.4907408

Nguyen, M. A., \& Zaghloul, A. I. (2007). On the Characterization of Cochannel Interference in an Aeronautical Mobile Environment. IEEE Transactions on Vehicular Technology, 56(2), 837-848. http://dx.doi.org/10.1109/TVT.2007.891440

Oudah, A. (2013). Resource Element-Level Dimensioning of Long Term Evolution Networks. Journal of Information and Communication Technology (JICT), 12, 189-205.

Oudah, A., Rahman, T. A., \& Seman, N. H. (2012). Coexistence and Sharing Studies of Collocated and NonCollocated Fourth Generation Networks In the $2.6 \mathrm{GHz}$ Band. Journal of Theoretical and Applied Information Technology, 43, 112-118.

Roke Manor Research Ltd. (2008). Practical Compatibility and Coexistence Measures Analysis, Hampshire, UK. 
Shamsan, Z. A., Rahman, T. B. A., \& Al-Hetar, A. M. (2012). Point-Point Fixed Wireless and Broadcasting Services Coexistence with IMT-Advanced System. Progress in Electromagnetics Research, 122, 537-555. http://dx.doi.org/10.2528/PIER11090305

3GPP TS 25.104, Technical Specification. "BTS Radio transmission and Reception (FDD)."

3GPP TS 25.942, Technical Specification. "RF System Scenarios.”

3GPP2, Technical Specification. "Recommended Minimum Performance Standards for cdma2000 Spread Spectrum BSs”, C. S0010-B, V1.0.

\section{Copyrights}

Copyright for this article is retained by the author(s), with first publication rights granted to the journal.

This is an open-access article distributed under the terms and conditions of the Creative Commons Attribution license (http://creativecommons.org/licenses/by/3.0/). 